\title{
EXPERIENCIA DE TRABAJOS ARQUEOLÓGICOS en zONAS de Selva Sur, en el Sector HIDROCARBUROS: MetOdOLOGÍA UTILIZADA EN EL LOTE 88 Y LOTE 56
}

\author{
Lic. Violeta ChamorRo
}

E ste trabajo pretende destacar que es perfectamente posible el desarrollo tecnológico en territorios de comunidades nativas, zonas de reserva territorial y áreas con presencia de sitios arqueológicos, siempre que se lleve a cabo un plan previo, durante y al finalizar los trabajos.

Así también, conocer lo referente la metodología que las empresas de hidrocarburos desarrollan técnicamente en las diferentes etapas de desarrollo de estas actividades de trabajo; básicamente queremos abordar los puntos críticos y además desconocidos cuando tenemos que insertar los trabajos de búsqueda e identificación de sitios arqueológicos, pues cuando queremos enmarcar nuestro trabajo en zonas de amazonía este trabajo resulta muy delicado y peligroso por la densa vegetación que no permite visualizar sitios, además de tener áreas inaccesibles.

This work emphasizes that the technological development is perfectly possible in native communities, territorial reserves and areas with the presence of archaeological sites, whenever a previous plan is carried out, during and at the end of the work.

In this sense, I will deal with the methodology that the hydrocarbon companies develop technically in thein activities and basically I will discuss the critical points of the research and identification of archaeological sites in these conditions in the Amazonian jungle. 
Nuestra experiencia de trabajos arqueológicos en zonas de Selva Sur, está basado en 5 años de labor continua de monitoreo arqueológico en todas las etapas de trabajo en el Lote 88 y Lote 56 adjudicados a la empresa Pluspetrol. Estos lotes se ubican en la región Sudeste del territorio peruano, en la vertiente oriental de la Cordillera de los Andes, Cuenca del río Urubamba, perteneciente al distrito de Echarate, provincia de La Convención, departamento del Cusco.

Este trabajo pretende destacar que es perfectamente posible el desarrollo tecnológico en territorios de comunidades nativas, zonas de reserva territorial y áreas con presencia de sitios arqueológicos, siempre que se lleve a cabo un plan previo, durante y al finalizar los trabajos.

Así también, insertar sobre todo la metodología que las empresas de hidrocarburos desarrollan técnicamente en las diferentes etapas de desarrollo de estas actividades de trabajo; básicamente queremos abordar los puntos críticos y además desconocidos cuando tenemos que insertar los trabajos de búsqueda e identificación de sitios arqueológicos.

Si bien es cierto que hay metodología y técnicas de trabajo arqueológico para los sitios conocidos, donde es posible su identificación con tan sólo visitar el área, como son las zonas de sierra o costa; sin embargo, cuando queremos enmarcarnos en zonas de selva este trabajo resulta muy delicado y hasta peligroso por la densa vegetación que no permite visualizar sitios, además de tener áreas inaccesibles.

La selva, debido a sus características climáticas, edafológicas y geográficas presenta un ecosistema complejo y heterogéneo, por lo cual los trabajos que se desarrollan deben tener una particular estrategia y logística adaptada a lo agreste e inaccesibilidad del área amazónica.

\section{Antecedentes}

Para abordar los temas de interés es necesario conocer los antecedentes de las empresas que trabajaron en la zona del Bajo Urubamba y así desarrollar las etapas de trabajo que estas empresas ejecutan en sus operaciones normales.

En el Perú, específicamente en el Bajo Urubamba, la actividad hidrocarburífera se desarrolla en una primera etapa entre los años 1983 y 1998, con Shell; en 1996 con Chevron; 1998 y 1999 la compañía Phillips Petroleum.

El Proyecto Gas de Camisea tuvo una primera etapa durante el primer quinquenio de los ochenta con la Compañía Shell, quienes se retiraron a mediados de la misma década; en 1996 la misma compañía juntamente con Mobil firmaron un acuerdo con PeruPetro para realizar actividades de perforación exploratoria preliminar en el bloque $88 \mathrm{~B}$ y analizar las opciones para la futuras exploración de 
las reservas de gas y líquidos de gas natural de Camisea ${ }^{1}$. Sin embargo, dos años después Shell se retiró al comunicar el gobierno peruano que no continuará con las operaciones.

Durante el gobierno de transición de Valentín Paniagua en el 2000, se firma el nuevo contrato con el consorcio formado por varias empresas: Pluspetrol, Hunt Oil Company Peru, SK Corporation Sucursal Peruana y Tecpetrol del Perú. El ámbito de concesión son los actuales Lote 88 y Lote 56. Adicionalmente el gobierno otorgó el contrato de licencia para el rubro de transporte a la compañía Transportadora de Gas de Perú (TGP), encargada de construir el gasoducto hasta la costa.

Nuestra experiencia de trabajo se inicia a partir del año 2001 con la empresa Pluspetrol, quien tiene en concesión los Lotes 88 y 56 ubicados ambos en el Bajo Urubamba. Estos lotes se ubican en áreas de comunidades nativas Matshiguenkas (Cashiriari, Segakiato, Shivankoreni, Camisea, Kirigueti, Nueva Vida, Mayapo y Puerto Huallana), una zona de reserva (Nahua - Kugapakori) y dos asentamientos de colonos (Shintorini y Malvinas).

Las operaciones desarrolladas en el Lote 88 fueron de exploración sísmica 3D, construcción con la Planta de Gas Malvinas, tendido de líneas de Conducción (FlowLine) que une la planta con los pozos San Martín 1 y San Martín 3.

En el Lote 56 se desarrolló exploración sísmica 3D, 2D, y actualmente se está trabajando en el tendido de líneas de conducción para llegar hasta la plataforma del Pagoreni, además de unir esta con la Planta de Gas Malvinas y la perforación de 12 pozos direccionales a partir de tres plataformas.

\section{El Medio Ambiente}

\subsection{Geomorfología y Geología}

Desde el punto de vista geomorfológico, el área de estudio pertenece a la llanura amazónica, caracterizada por presentar superficialmente un relieve levemente ondulado, que se distingue por el despliegue de superficies relativamente planas, lomadas y colinas de poca altura, que descienden hacia los cauces de los ríos. La superficie de estas terrazas está conformada por sedimentos constituidos por limo, arcilla y arena. Los estratos observados en las calicatas están constituídas por sedimentos relativamente profundos del cuaternario, a base de material arenolimoso y arcillas, dispuestos en terrazas de diferentes alturas, desde altas hasta bajas. En el plano aluvial, se observa la presencia de playas con deposiciones limosas, la mayoría de ellas constituidas por cantos rodados.

Estudio de Impacto Ambiental, ERM, 2005, p. 4. 
En general, la llanura amazónica constituye una depresión estructural rellenada predominantemente con depósitos de material clásico de origen continental. El proceso geológico a través del cual se produce la deposición del material de relleno, tiene su origen en la forma en que los ríos van construyendo su cauce.

El cauce sinuoso, típico de los ríos amazónicos, que discurren formando una permanente sucesión de meandros, en la cual los tramos rectos son casi inexistentes, produce un permanente proceso de erosión y deposición de los sólidos transportados por el río, los cuales están compuestos tanto por material que es arrastrado desde las partes altas, principalmente durante las temporadas de lluvia, como por los materiales desprendidos de zonas más cercanas como consecuencia de la erosión.

Subyaciendo los depósitos del cuaternario, se encuentran formaciones que corresponden a depósitos del terciario, representados por areniscas poco consolidadas y de escasa dureza, cuya presencia es claramente apreciable en distintos puntos de la ribera, tanto en la zona misma del proyecto, como aguas arriba y debajo de la zona².

\subsection{La vegetación}

En el área de estudio se distinguen distintos tipos de formaciones vegetales que poseen una compleja y muy diversa composición florística. Básicamente se reconoce dos paisajes de vegetación, uno natural conocido como Bosque Primario que conserva un ecosistema en estado virgen, boscoso y maduro, el cual no ha sido alterado por la intervención humana.

El otro tipo de vegetación es conocido como Bosque Secundario es alterado e intervenido con una cubierta vegetal de cultivos y comunidades vegetales de regeneración o "Purmas". Estas «Purmas» son el resultado de la regeneración natural del bosque cuando una parcela agrícola es abandonada por el agricultor debido al agotamiento de la fertilidad natural del suelo. La parcela así abandonada al cabo de varios años es ocupada nuevamente por el mismo $u$ otro agricultor.

Se observan tres etapas de sucesión secundaria en los bosques. La primera consiste en matorral, con arbustos, herbáceos y lianas; la segunda con especies de crecimiento rápido como la Cecropia (Fabaceae), Ochroma (Bombacaceae), Trema (Ulmaceae), Vemonia (Asterraceae); y la tercera cuando el bosque tiene más de 8 metros de altura. La vegetación está conformada por el bosque ribereño, pastizales y el bosque de terrazas con pacales (Guadua sarcocarpa), éstas últimas son áreas dominadas por cañas de bambú, con predominio sobre la vegetación arbórea, los que se presentan esporádicamente, caracterizados por un menor desarrollo tanto en altura como en diámetro.

2 Proyecto Monitoreo Arqueológico Lote 56, p.3. 
«He mencionado los tipos de bosques por una razón importante, pues el arqueólogo al diferenciar el tipo de bosque, también va a observar con mayor detalle su entorno cuando realiza los recorridos y acompañamientos a los grupos de trabajo que ingresan al monte.

Por mi experiencia puedo mencionar que, durante mi recorrido y acompañamiento en la apertura de trochas, pude verificar que el tipo de bosque secundario, acompañado con terrazas planas y sobre todo con presencia de pacales, es un lugar potencial para detallar mejor nuestra observación; en estos espacios hay que tener mucho cuidado en la identificación; cabe recordar que en un primer momento no se aprecia nada a simple vista que nos permita verificar presencia cultural; sin embargo, se recomienda buscar en los árboles caídos. Si es un lugar con restos arqueológicos observará fragmentos de cerámica que se desprenden de las raíces, así también se debe buscar en los hoyos que los animales hacen y acumulan tierra, aquí también encontrará fragmentos dispersos» ${ }^{3}$.

\subsection{Vías de Acceso a los Lotes 88 y Lote 56}

El acceso es directamente por vía aérea desde Lima al Campamento Base Malvinas; por vía terrestre se va desde Lima a la ciudad de Pucallpa en el departamento de Ucayali, continuando por vía fluvial hasta el distrito de Sepahua y desde allí hasta el poblado de Nuevo Mundo ubicado en el Lote 56 y el Campamento base Malvinas en el Lote 88, teniendo en esta zona como principales ríos de navegación el Urubamba y el Camisea.

\section{Importancia del Estudio de Impacto Ambiental (EIA)}

Toda empresa de hidrocarburos a quien PERUPETRO le adjudica un lote para ser explorado y explotado, inicia su trabajo con el Estudio de Impacto Ambiental (EIA), estudio que se realiza a través de una empresa consultora independiente.

Los resultados de este estudio lo presenta la empresa adjudicataria del lote, éste puede ser para exploración o explotación de hidrocarburos al Estado Peruano, específicamente al Ministerio de Energía y Minas (Dirección General de Asuntos Ambientales Energéticos, DGAAE/MEM), el cual debe ser aprobado y así solicitar los permisos correspondientes; aquí se tienen en cuenta todas las leyes peruanas para los diferentes rubros, sea medio ambiente, estudios sociales como trabajos en comunidades nativas, zonas de reserva y sitios arqueológicos.

Este es el punto de partida del trabajo del arqueólogo, mediante la presentación de un proyecto de reconocimiento arqueológico al Instituto Nacional de Cultura (INC), con fines de solicitar el CIRA (Certificado de Inexistencia de Restos Arqueológicos) del Lote a evaluar en la preservación del patrimonio cultural, pues

Comentario de Violeta Chamorro. 
su participación se da bajo la modalidad de prospectar áreas e identificar sitios arqueológicos y así poder tramitar el documento que emite el INC como CIRA.

En el proceso de trabajo para obtención del CIRA, si el arqueólogo identifica sitios arqueológicos debe presentar un proyecto al INC donde emite resultados y estrategias a continuar, por ello es importante conocer todas las etapas de trabajo donde se debe considerar varios puntos a desarrollar.

Inicialmente el arqueólogo se inserta a la empresa que realiza el EIA, y sus resultados y recomendaciones los emite para zonas puntuales, más no para el lote completo, dentro de estas recomendaciones es necesario considerar que durante los trabajos de obras civiles y sísmicas debe considerase el monitoreo arqueológico permanente, el cual es la línea base del supervisor del INC, cuando ingresa a verificar los trabajos del arqueólogo.

Se piensa que con la expedición de CIRA, por parte del INC se terminó la labor del arqueólogo y que no hay nada que hacer en las actividades de operaciones de las empresas que operan en hidrocarburos, grave error, pues es aquí donde se inicia el trabajo y el arqueólogo debe conocer todas las etapas a ejecutarse en la zona donde ingresó a trabajar, para planificar cada estrategia en el proyecto que debe presentar al INC, sobre el monitoreo arqueológico.

De todas las etapas mencionadas en los trabajos que la empresa Pluspetrol planificó, la de exploración sísmica fue la más importante para el arqueólogo, pues aquí se tuvo la oportunidad de tener toda la logística adecuada para poder ingresar a la zona, sobre todo porque la sísmica involucra el ingreso de mucha gente a las áreas que nos interesa prospectar y/o monitorear.

Las demás etapas del trabajo se van a complementar con los primeros resultados que arroje el monitoreo arqueológico en sísmica, pues aquí se tendrá una primera visión del área y de la posible ubicación de sitios arqueológicos. Para ello es necesario conocer en qué consiste una sísmica y cómo los arqueólogos debemos insertarnos en estas operaciones.

\section{La Exploración Sísmica}

La exploración sísmica es uno de los estudios realizados durante el proceso de búsqueda de hidrocarburos como petróleo o gas. Este estudio dura un promedio de tres a cinco meses aproximadamente, dependiendo de los registros que necesiten las empresas, bien de 2D o de 3D.

Antes de escoger un área para la exploración sísmica, un equipo de geólogos y geofísicos ya han estudiado mapas geológicos, fotografías aéreas o imágenes satelitales, además de visitar el terreno o sobrevolarlo; el objetivo es que sirvan para detectar zonas con posibilidades de contener yacimientos de gas o petróleo. 


\subsection{Métodos utilizados en los estudios previos}

\subsubsection{Gravimetría y Magnetometría Aéreas}

Se trata de un método donde se obtiene información relacionada con las propiedades de las rocas, tales como densidad y características magnéticas. Esta información se adquiere con el sobrevuelo de una pequeña aeronave, la cual lleva a bordo instrumentos especializados. Los estudios permitirán conocer mejor la geología del sector y facilitarán el diseño de otras actividades, como la adquisición sísmica que busca información más detallada. Esta actividad puede durar entre dos y tres meses, dependiendo de las condiciones climáticas. ${ }^{4}$

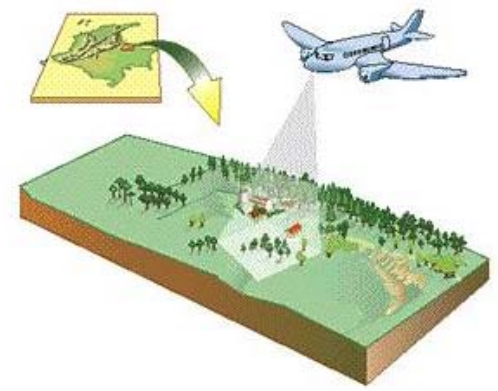

Figura 1.

Gravimetría y Magnetometría Aérea

\subsubsection{Fotografías aéreas digitales de alta resolución}

Como su nombre lo indica, son fotografías de alta resolución tomadas desde un pequeño avión, que permitirán obtener una imagen en tres dimensiones del terreno y serán herramienta fundamental para la realización de estudios ambientales, geológicos y geofísicos. De comprobarse la presencia de hidrocarburos que se puedan producir de manera comercial, estas imágenes serán de gran ayuda para el diseño de estudios geotécnicos y de infraestructura en general. ${ }^{5}$

\subsubsection{Estudios geológicos de campo}

En esta actividad un grupo de geólogos recorre algunos sectores de la región, como caminos, carreteras y quebradas, tomando muestras de las rocas, para luego ser estudiadas más al detalle en laboratorios. Este estudio es un complemento de todas las anteriores actividades, como resultado se tiene un mejor conocimiento de la distribución de las diferentes capas geológicas en la región y sus características, que entre los geólogos se conoce como el mapa geológico. ${ }^{6}$

ECOPETROL, datos tomados de Internet 04.05.06.

Ibid.

Ibid. 


\subsection{Tipos de Exploración Sísmica}

4.2.1 Sísmica 2D.- Se denomina sísmica 2D porque la adquisición de datos solo se hace en dos dimensiones, pero mirado desde el punto de vista espacial, es decir se graba información solo en un sentido en superficie, más la variable profundidad; de este modo se recuperan datos en dos dimensiones. La adquisición de datos en sísmica 2D consta de un pattern de adquisición (números de canales grabados) y la fuente de energía distribuidos solamente en la dirección de orientación de la línea que se está registrando. Los puntos «fuente de energía» pueden ser pentonita en selva, vibradores en zonas abiertas o aire comprimido utilizado principalmente en mar.

En una línea 2D en selva se usa pentonita como fuente de energía, en una línea con receptoras y en la misma línea cada cierta distancia las cargas de los puntos explosivos. Aquí se adquiere información solo en la dirección de esa línea ubicada de Norte-Sur y Este-Oeste o la dirección que tenga esa línea en particular. Estos puntos se distribuyen a lo largo de la línea receptora con un espaciamiento entre ellos de acuerdo al diseño geofísico requerido para la información que se pretenda conocer; de igual forma acontece con la ubicación de los sensores o receptores (geófonos) que convertirán la información acústica en señal eléctrica para ser procesada en equipos electrónicos de alta resolución.

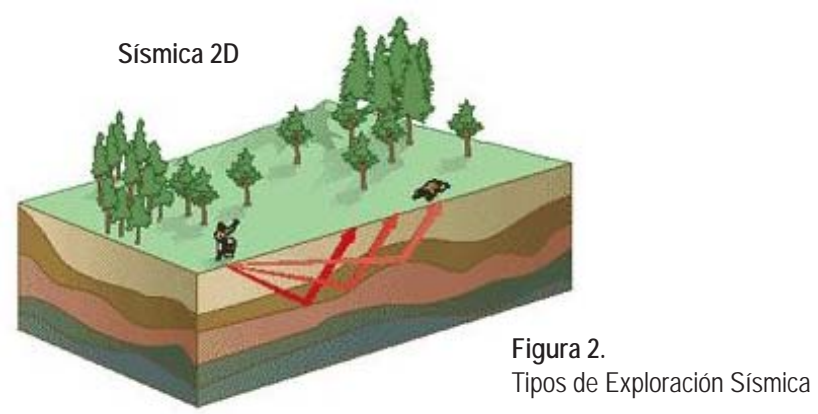

La apertura de las líneas 2D sigue el diseño del geofísico y el proyecto recibe el nombre 2D porque la adquisición de datos solo se hace en la línea que tiene los puntos de disparo y se graba solo con los receptores ubicados en la misma línea. ${ }^{7}$

4.2.2 Sísmica 3D.- Siguiendo la misma metodología en lo referente a fuentes de energía y plantado de receptores aplicada en la sísmica 2D, en la sísmica 3D se adquiere información en tres sentidos: Profundidad y dos variables en superficie; una de las variables de superficie es en el sentido de la orientación del plantado de los receptores

Datos proporcionados por el Company Man del Proyecto Sísmica 2D y 3D Pluspetrol, durante la exploración sísmica del Lote 56 en el año 2005. 
para cada una de las líneas que forman la geometría del diseño de adquisición (template) y la otra variable de superficie se obtiene de la información que se graba en las líneas paralelas que conforman el «template» de adquisición. Para comprender mejor se debe tener en mente que en un proyecto 3D se adquiere información sísmica de varias líneas receptoras al mismo tiempo $(4,6,8,10$ o más) utilizando un punto común como fuente de energía (punto de disparo) y en un proyecto $2 \mathrm{D}$ solo se graba la información en una línea receptora individual; los datos de un 3D se obtienen de un rectángulo y en el 2D se graba información solo de una recta. ${ }^{8}$

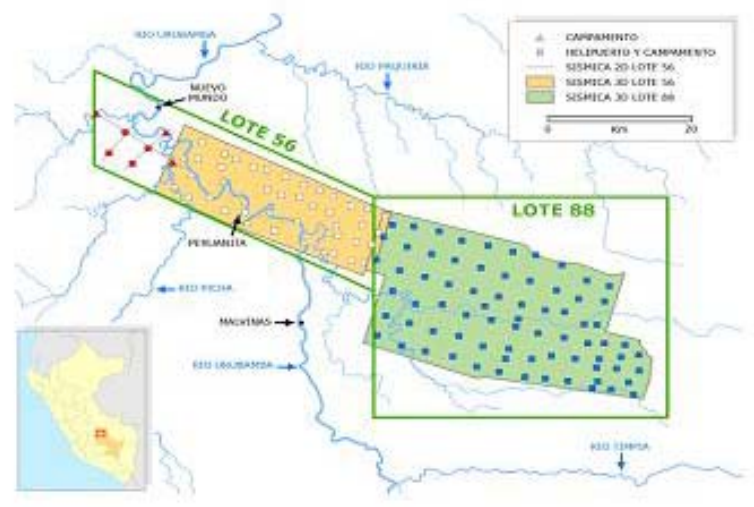

Figura 3.

Plano de Ubicación de los Lotes 88 y 56; Pluspetrol es la empresa aperadora.

\subsection{Etapas de la Exploración Sísmica}

Antes de iniciar el programa sísmico se realizan estudios ambientales para identificar el estado de los recursos naturales (componente físico y biológico como es el agua, suelo, aire, vegetación, fauna) y la situación actual del área. Se analizan las formas en las cuales el proyecto podría afectar el medio ambiente, identifica el capital humano, físico y económico y sobre todo la población involucrada dentro del proyecto como pueden ser comunidades nativas y caseríos o la probabilidad de afectar sitios arqueológicos y encontrarse con problemas sociales. Se toman medidas requeridas para prevenir, controlar y mitigar los efectos negativos y se cuantifican los efectos positivos.

En la exploración sísmica se tienen 3 etapas de trabajo: Topografía, Perforación y Registro.

Ibid. 


\subsubsection{Instalación de campamentos y helipuertos}

La ubicación y cantidad de campamentos y helipuertos (HP) ${ }^{9}$ depende de varios factores como: requerimientos operativos, facilidades de acceso y tipo de equipamiento utilizado en la operación; pero lo más importante es que se ubican cerca de puntos de agua, los que pueden ser ríos o quebradas. La distancia promedio entre los HP es de 2.5 a 3 kilómetros.

Los campamentos pueden ser de diversos tipos, dependiendo de su capacidad. Un campamento base, se instala preferiblemente en un área poblada, desde donde se coordinan los trabajos. En este lugar se aloja el personal técnico y los equipos, se adecúan talleres, bodegas, oficinas, baños, pozos sépticos y fosas de basura, cumpliendo con las medidas de conservación ambiental y las regulaciones existentes.

Se instalan también campamentos «volantes» en áreas retiradas, con el objetivo de evitar largos desplazamientos; estos deben ser preparados previamente por un primer grupo que ingresa a las áreas de trabajo (llamados campamenteros), quienes despejan el área y la adecuan para instalar el campamento volante que consta de un espacio de $20 \times 20 ; 30 \times 30$ y/o $40 \times 40$ metros aproximadamente, este campamento debe considerar cocina, comedor, dormitorios, etc. Aquí se realizan algunos zanjas como: pozos sépticos, trampas de grasa para contener residuos alimenticios (cocina), jabón de las duchas y lavandería de ropa, así también se realizan remociones en el área de combustible y donde se ubica el generador o en la habilitación de escalinatas tanto en los campamentos como en las líneas.

Cerca al campamento, también se apertura en un área plana y elevada un espacio para ser utilizado como helipuerto, para poder transportar los materiales como alimentos, equipos, personal mediante helicópteros; por ello son conocidos como HP (helipuerto). Los campamentos son enumerados correlativamente con números arábigos del 1, 2, 3 hasta los planificados en el plano y los aceptados en el EIA. Por lo tanto para identificar cada campamento se denomina por ejemplo HP1, HP2, HP3 y así sucesivamente.

En el caso de llevarse a cabo una sísmica 2D, de manera continuada a la sísmica 3D, como se realizó en el Lote 56, para evitar confusión, los campamentos son enumerados de acuerdo a la nomenclatura internacional conocida como: Alfa, Bravo, Charly, Delta, Eco, Fox y Golf para identificar los campamentos volantes utilizados como apoyo logístico a las operaciones.

$9 \quad \mathrm{HP}$, es la identificación de los helipuertos, pocas veces se instalan solos, siempre están asociados a un campamento volante. 


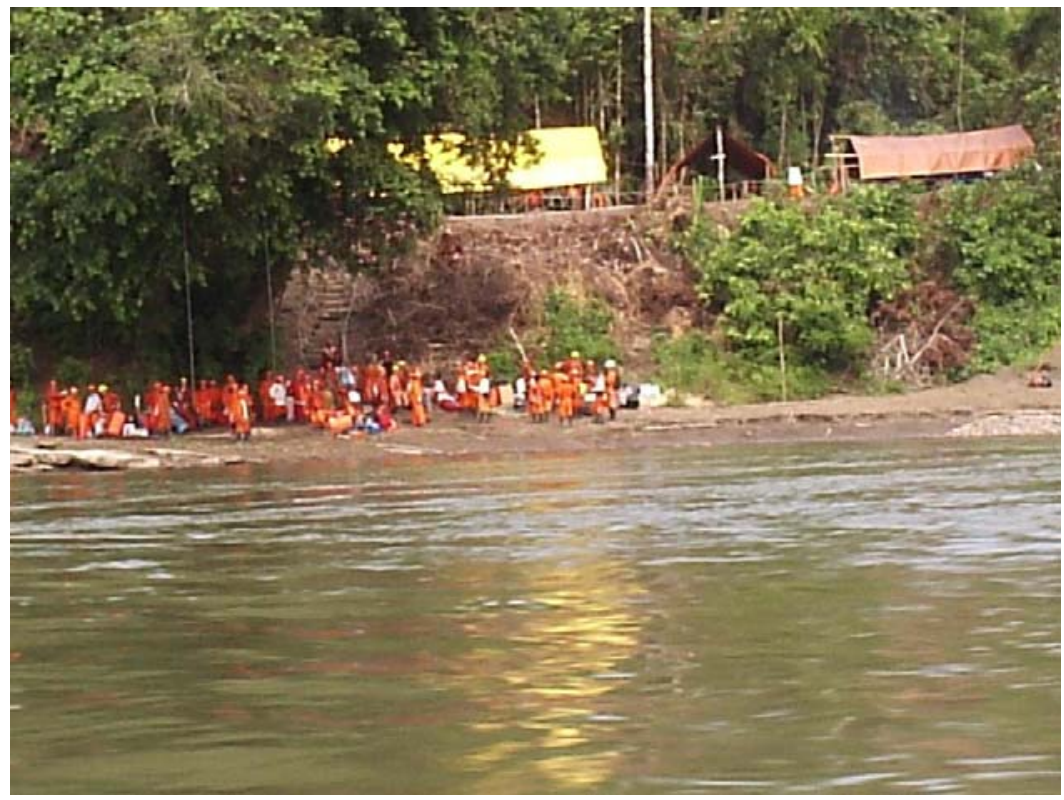

Figura 4.

Campamento Volante 35 (Lote 56)

Luego de tener la primera habilitación del área, ingresan los grupos de trabajo para la etapa de topografía, equipo compuesto de 3 topografías. Estos lo conforman un topógrafo y 14 trabajadores en la zona, por lo tanto estarían ingresando un promedio de 45 personas.

\subsubsection{Etapa de topografía}

El ingreso de la topografía es la primera etapa de trabajo en una exploración sísmica, en esta etapa se ejecuta el trazado de las líneas sísmicas, mediante un levantamiento topográfico (líneas emisoras o fuentes y líneas receptoras) ${ }^{10}$. Este se realiza con equipos de alta precisión; las cuadrillas ( 45 en total) utilizan como punto de inicio georeferenciado la red de puntos GPS localizados en cada una de las áreas

10 Línea emisoras o fuentes, también la conocen como líneas de salvo. La dirección de la línea de salvo está compuesta por varios puntos perforados llamados estaciones fuentes, estos puntos perforados son cargados con material explosivo, tipo de material que depende de la empresa que realiza el trabajo, para el caso del Lote 88 y Lote 56, se utilizó material explosivo biodegradable conocido como pentonita. La dirección de estas líneas es de Sur o Sureste a Norte o Noroeste. Líneas receptoras es la destinada para colocar los cables los cuales tiene, las ristras o geofonos los cuales son incrustados bajo tierra en cada punto, por medio de estos actúan como sensorias al momento de realizar los disparos y así se puede registrar la áreas que contienen gas o petróleo. 
que se abren como apoyo logístico a las operaciones. Esta etapa permite georeferenciar el proyecto de acuerdo al diseño de coordenadas previamente establecidas. Estos puntos de GPS se ubican en cada campamento volante, los cuales se identifican con un testigo permanente consistente en un poste y una placa metálica donde se señala su identificación. Estos puntos de GPS son importantes para georeferenciar los hallazgos culturales registrados.

Las cuadrillas se encargan de aperturar las trochas, si es boscosa se desbroza un espacio de 1.20 metros; si la vegetación es escasa, como rastrojo o maleza, será necesario aperturar 1 metro de ancho, aquí se traza la dirección que debe seguir la topografía. Al mismo tiempo se señalizan las líneas trazadas mediante estacas y/o banderas. Las trochas serán temporales y se harán todos los esfuerzos posibles para devolverlas a su condición original, se realizan esfuerzos para minimizar la tala de árboles con diámetro no mayor de 0.10 a $0.20 \mathrm{~m}$.

El topógrafo utiliza teodolito, prisma y jalones para medir distancias, ubicar puntos de pozo y marcar los intervalos. Con este estudio se obtiene un perfil del terreno. En esta etapa además de aperturar la línea sísmica se apertura o desbroza en cada cruce de receptora con línea de disparo un drop zone, conocido como DZ, el cual es de $4 \times 4 \mathrm{~m}$. El DZ, es importante porque sirve para dejar -utilizando una línea larga-, los cables y ristras que serán regados en la línea.

La apertura de trocha o desbroce, se realiza en forma manual con machete y motosierra, se abre una línea de $1.50 \mathrm{~m}$. de ancho como máximo.

Esta es la etapa más importante del monitoreo arqueológico, pues consiste en realizar el acompañamiento a los diferentes grupos de topografía, al momento que deben realizar la apertura de las líneas sísmicas, con el objetivo de verificar en el campo la presencia o ausencia de restos culturales. En este recorrido se va monitoreando las líneas receptoras y las líneas donde se ubican los puntos de disparo o de salvo, así también se realizan variantes que son rutas ubicadas fuera de las líneas sísmicas, pero que ayudan a buscar rutas más accesibles para que los grupos siguientes no tengan mayores problemas de acceso, en cada cruce de una línea de disparo y una línea receptora se realizan desbroces en un espacio de $4 \times 4 \mathrm{~m}$. conocidos como zonas de descarga (Drop Zone «DZ»), en estos espacios el helicóptero deja material de trabajo. En el campo se identifican estas líneas por la numeración asignada anticipadamente por ejemplo (6151/stk1646) y los DZ son denominados con letras alfabéticas.

Es importante conocer que todo punto en el terreno está georeferenciado, lo que permite tener la ubicación exacta de los sitios culturales registrados. 

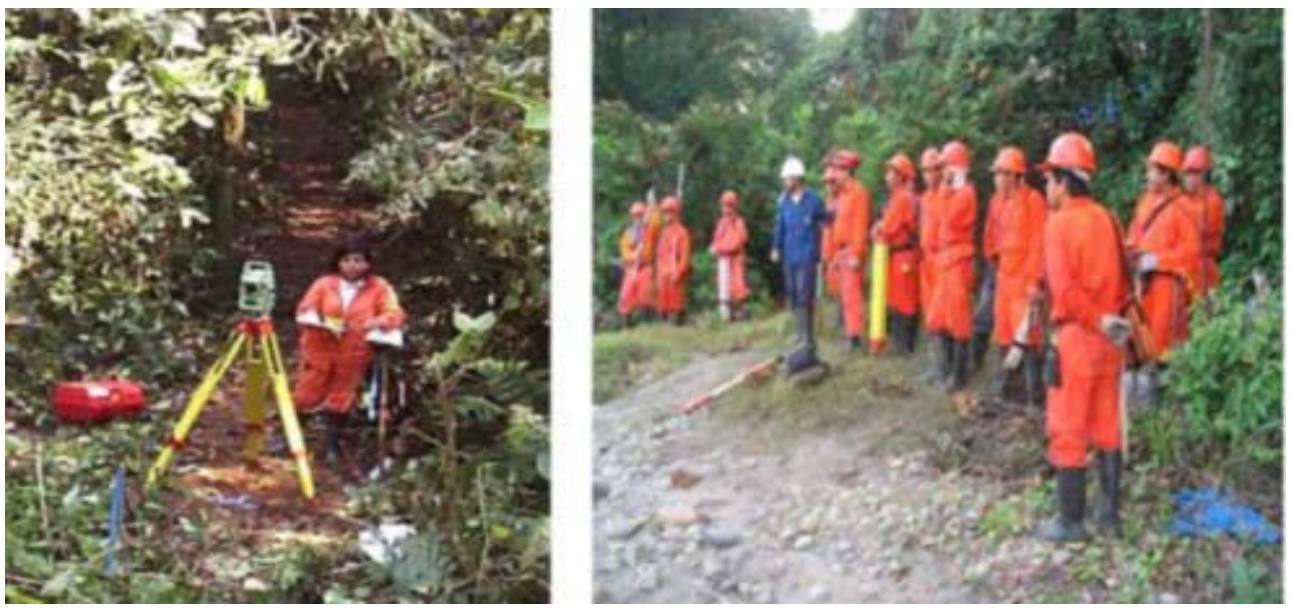

Figura 5 y 6 .

El grupo de topografía que ingresa en la primera etapa de trabajo, abre las líneas sísmicas.

\subsubsection{Etapa de taladro}

Cuando en la etapa de topografía se tiene avanzado un promedio aproximado del 30\% de trabajo empiezan a ingresar los grupos que conforman la perforación a cada campamento volante que abandonó el grupo de topografía y las líneas sísmicas ya están abiertas.

Estos grupos de perforación, abren pequeños orificios de 0.10 a $0.15 \mathrm{~m}$. de diámetro en cada estaca de las líneas fuentes o salvo, con un instrumento llamado carey, taladrando una profundidad de $15 \mathrm{~m}$. de diámetro, o repartidos estos hoyos en 3 ó 5 en caso no sea posible perforar a la profundidad estándar.

Las líneas trazadas tienen estacas espaciadas a 40 metros para colocar carga en el fondo del hoyo, este hoyo luego es tapado para que el geófono reciba mejor la vibración; estas distancias varían para el cuidado ambiental: cuando se ubican en fuentes de agua y vivienda la distancia es de 100 metros, y en bocatomas de acueductos $150 \mathrm{~m}$.; el registro de sitios arqueológicos también tiene estos parámetros de distancia para evitar deteriorar los sitios

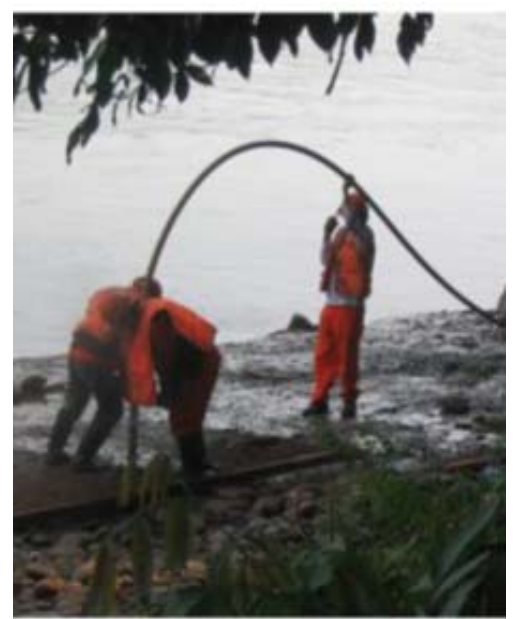

Figura 7.

Perforando utilizando el taladro arqueológicos. 


\subsubsection{Etapa de regado y registro}

Mientras que en la etapa de topografía se está por concluir la apertura de líneas, y en la etapa de taladro se avanzó en un 50 \% aproximadamente, ingresa el personal que debe realizar el trabajo de regado y registro.

Esta etapa de registro, consiste en un grupo que coloca sensores en movimiento (geófonos) a través de toda la línea sísmica, estos sensores son muy sensibles y cuando ya se tiene todo listo y conectado mediante cables al equipo de computación que procesa los datos (Sismógrafo) procede a mandar a los disparadores, grupo de especialistas que conecta las cargas de un pozo previamente perforados. Una vez listos avisan al sismógrafo, ubicado en el punto desde donde se explotan las cargas.

La pequeña carga que ha sido puesta en el fondo de cada hoyo es activada con el fin de producir unas ondas que viajan por el subsuelo reflejándose en diferentes tipos de rocas hasta llegar nuevamente a la superficie. Estas vibraciones son detectadas por pequeños geófonos que transportan la información a un equipo de registro donde posteriormente es procesada y analizada por expertos.

Los geófonos, cables y demás equipos son inofensivos; el personal que maneja la carga es altamente calificado para este tipo de trabajo.

La interpretación de los resultados se dan una vez recogidos los datos por los equipos, viajan por señales de radio al campamento base, donde los expertos analizan e interpretan los resultados.

Una vez hecho y grabado un disparo, los disparadores pasan al pozo siguiente y así sucesivamente hasta terminar el registro total de una línea.

Posteriormente los obreros recogen el material (cables y sensores) y continúan en la línea siguiente, y así sucesivamente hasta terminar el proyecto.

Cada vez que se va recogiendo el material de línea, otro grupo de medio ambientalistas va recogiendo todo el material o basura arrojada en las líneas y tapando o sellando algún pozo mal detonado si es que se da el caso, de igual forma no es problema porque no causa daño y el material se degradará en su momento.
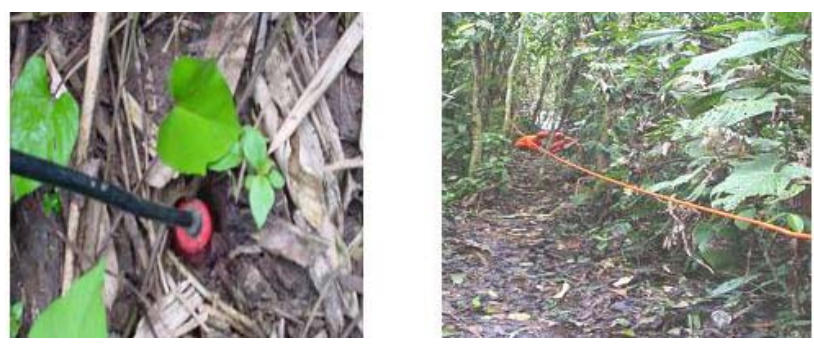

Fuguras 8 y 9.

Geófono colocado en líneas receptoras.

Cable de línea sísmica o ristra. 


\subsubsection{Plan de abandono y reforestación}

Culminadas las tres fases de trabajo tenemos el plan de abandono, el cual consiste en dejar las áreas intervenidas limpias de basura y habilitadas para poder ser reforestadas.

Aquí se retira todo elemento considerado como basura y se cubre de ramas, palos o cualquier vegetal en la superficie, mientras que anticipadamente, un grupo de trabajadores conocidos como el grupo verde, compuesto por un ingeniero forestal y personal de apoyo, arman viveros con plantas de la zona, las cuales son transplantadas en toda el área de campamento y helipuertos. Para el caso de las líneas sísmicas abiertas, no es necesario reforestar porque este tipo de vegetación tiende a regenerarse en poco tiempo y las líneas sísmicas se cierran muy rápidamente.

\section{Etapa de trabajo de construcciones}

\subsection{Las plataformas de perforación}

Las plataformas han sido diseñadas y están en proceso de construcción. De acuerdo a las características del equipo de perforación se utiliza la normatividad vigente y aspectos como las condiciones del terreno colinoso, el campamento, la zona de servicios, la plataforma de aterrizaje de helicópteros. La plataforma de perforación tendrá el espacio para alojar los depósitos de química para el lodo y cemento, depósito de equipos, depósito de agua para la perforación, equipo de tratamiento y control de sólidos, quemador, área de tratamiento de desechos y descargas, área de viraje, depósito de diesel, entre otros.

Las obras civiles que se han ejecutado comprenden lo siguientes aspectos:

- Deforestación del área, utilizándose los troncos para la construcción de las distintas obras, como estabilización de taludes, estabilización de accesos temporales o caminos peatonales.

- Movilización y desmovilización de equipos y materiales.

- $\quad$ Armado y desarmado de equipos, para el movimiento de tierras.

- Habilitación de un campamento temporal para la etapa de construcción.

- Construcción y acondicionamiento de la plataforma, lo cual constituye entre otras obras, el corte, perfilado, nivelación, compactación, conformación de drenaje interno y externo de la misma y de todas las áreas anexas.

- Construcción de la fosa de las pruebas sísmicas.

- Instalación de trampas de aceites y grasas. 
Para todas las instalaciones en superficie se realizan excavaciones localizadas para la función de los equipos. El excedente de corte de terreno es dispuesto en los botaderos de cada locación y parte de los recortes útiles serán usados como aporte para el recinto superior de la fosa de recortes de perforación.

Los drenajes de agua construidos están dirigidos hacia las trampas de aceites y grasas. El agua de lluvia es conducida directamente a los canales externos de la plataforma para ser tratada de manera preliminar en las trampas y ser descargada en superficie a un cuerpo de agua próximo.

El monitoreo arqueológico en esta zona se realiza permanentemente dado que existen grandes movimientos de suelos, para hacer el terreno asequible para construir la plataforma.

\section{Metodología y estrategias de trabajo arqueológico en el Sector}

\subsection{Durante el Estudio de Impacto Ambiental (EIA)}

En este tema abordaremos la metodología para monitorear y ubicar sitios arqueológicos y/o culturales.

Iniciaremos mencionando que el primer punto de este trabajo es cuando se está ejecutando el EIA, momento en que el arqueólogo forma parte del staff de profesionales; es muy importante el trabajo en equipo sobre todo con el geólogo, el botánico o forestal y el topógrafo, orientando la planificación del trabajo al caminar con ellos.

El geólogo realiza pequeños zanjeos para retirar muestra de suelos, es precisamente en ese momento que el arqueólogo debe estar atento en verificar si los pequeños hoyos contienen evidencias. En el Lote 56, en esta etapa de trabajo se registró el sitio cultural denominado Mipaya ${ }^{11}$.

El topógrafo realiza los primeros ingresos abriendo líneas bases y recorridos para ver los posibles trazos de las líneas de conducción, el recorrido de enlace de los pozos y campamentos. El acompañamiento al topógrafo es importante porque además de contar con toda la logística apropiada, permite verificar la presencia de evidencias en los tramos abiertos por los trocheros, y así la posibilidad de identificar áreas con presencia cultural; esta primera etapa nos permite dar soluciones acertadas antes que se generen problemas de paralización de maquinarias por ubicación de sitios arqueológicos. Así también, estos primeros tramos abiertos van a dar una idea del área a trabajar y del posible material a encontrar.

$11 \quad$ OP. Cit. 2004. P. 15. Durante la realización del EIA, para el Lote 56 la arqueóloga Rosa Marín reportó el hallazgo. 
Durante el EIA del lote 88, se registraron piedras talladas. ${ }^{12}$ Además permitió dimensionar y orientar nuestro trabajo durante el monitoreo arqueológico.

Los ingenieros forestales y botánicos nos permitirán conocer el entorno ecológico, los tipos de bosque; este punto es importante porque donde hubo ocupación humana el tipo de bosque es secundario y la vegetación es típica de estos bosques. Este tipo de bosques nos permitirá correlacionar áreas aterrazadas con presencia de pacales como posibles sitios conteniendo bajo tierra evidencias culturales, de modo que durante el monitoreo se tenga más precaución en la observación.

\subsection{Inicio de trabajo en los lotes adjudicados: charlas de sensibilización sobre patrimonio cultural}

Las charlas de inducción brindadas sobre patrimonio cultural son emitidas con presentación de transparencias y diapositivas, las denominamos inducciones y han permito sensibilizar sobre patrimonio cultural a jefes y a muchos trabajadores, quienes además proceden de otros departamentos de nuestro país, trabajadores de la zona y expatriados. Son importantes porque el personal que ingresa a trabajar tiene los conocimientos básicos sobre patrimonio cultural y sabe cómo actuar si se encontrase con estos hallazgos.

Estas charlas fueron las más importantes porque se orientó y explicó el trabajo del arqueólogo en la obra y la importancia de la recuperación de estos sitios arqueológicos. Esto nos permitió tener además de primera mano sitios intactos, los cuales son únicos, nunca fueron alterados por el ser humano (huaqueo), y se han mantenido intactos en el tiempo desde que sus ocupantes desaparecieron hace 300 años AC. Otro tema importante es la explicación sobre la función y supervisión que realiza el INC con respecto a la fiscalización de patrimonio, las leyes que protegen y las penalidades a las que estamos sujetos en caso no se cumpla con esta protección.

La mayor cantidad de trabajadores sensibilizados se dio al momento de realizar la exploración sísmica, para el lote 88 fueron 1600 personas y para el lote 56 se sensibilizó a unas 900 personas. La sísmica es uno de los trabajos donde ingresan mucha gente y la logística es apropiada; éste es el momento adecuado para ingresar a monitorear y así tener una idea general de lo que se encontrará durante los trabajos de obras civiles en la etapa de construcción.

Para el caso de Pluspetrol, además se generó dentro de su política un procedimiento a seguir en caso de ubicar sitios o evidencias culturales durante los trabajos.

12 Estudio de Impacto Ambiental, 2000, ERM. Durante la realización del EIA, en el Lote 88 reportó la arqueóloga Lucía Medina. 
- Paralizar los trabajos.

- Comunicar al Supervisor de Campo, en este caso el arqueólogo.

- Cercar el área con cinta para evitar más deterioro y no movilizar los objetos en caso sean visibles.

- El arqueólogo evalúa la importancia del hallazgo y ejecuta los trámites con el INC y los trabajos necesarios para liberar el área en el menor tiempo posible.

Cabe mencionar que durante los trabajos de sísmica donde se reportaron los mayores hallazgos y se ubicó los sitios arqueológicos fue por la toma de conciencia que se generó en cada persona que ingreso al área de trabajo.

Si la inducción está bien orientada, los resultados en los reportes y los procedimientos a seguir en caso de ubicar evidencias culturales dentro de áreas de trabajo es más productivo. Durante la sísmica del año 2002 en el Lote 88 , se realizaron reportes de hallazgos durante los trabajos de apertura de líneas sísmicas, la etapa de construcción, perforación y reforestación.

La toma de conciencia de los trabajadores, a veces también genera que se emitan reportes de lugares, con apariencia de presencia de restos culturales. Al evaluar un área nos damos cuenta de la confusión, como es el caso de dos reportes ejecutados en la sísmica del Lote 88 , sobre un posible camino, siendo este una simple hilada de piedras naturales, efectivamente daban la apariencia de ser un camino, pero en este caso fue solamente naturaleza. Otra anécdota es el reporte de vasijas en la dirección donde se debía aperturar una línea sísmica. Se realizó una caminata muy larga hasta llegar al lugar del reporte y efectivamente, a primera vista parecía una vasija de cerámica dispuesta boca abajo, pero al acercarnos se trababa de piedras redondas de color marrón oscuro generado por la oxidación y partidas por la mitad, siendo de origen natural. Sin embargo, si estos reportes son emitidos por el grupo de trabajo, siempre es importante explicar por qué no corresponde a una evidencia y emitir un agradecimiento especial por su apoyo en la recuperación del patrimonio cultural: Siempre es preferible tener reportes equivocados y verificar, antes de subestimar el reporte, pues siempre cabe la posibilidad que uno de los sitios reportados esté en lo correcto. Así nos permite dar soluciones inmediatas a cada caso presentado.

\section{3 ¿Cómo aprovechar la logística en cada etapa de trabajo durante el monitoreo arqueológico?}

\subsubsection{En la exploración sísmica}

Nuestra experiencia de trabajo en sísmica fue durante los trabajos en el Lote 88, ejecutado desde enero del 2002 a setiembre del mismo año, periodo en el cual se 
realizó una sísmica 3D, en un área de $1200 \mathrm{~km}^{2}$ y en el Lote 56 se trabajó sísmica 3D y $2 \mathrm{D}$ en un área de $580 \mathrm{~km}^{2}$.

Aquí hay que tener en cuenta que estas operaciones tienen un plan definido de tiempo de inicio y finalización del trabajo, costos y presupuestos, además de la planificación técnica.

Así mismo, la empresa que contrata los servicios en la exploración sísmica (en nuestro caso Pluspetrol) tiene un responsable experto en exploración sísmica (Company Man), jefe máximo y quien autoriza todos los trabajos de campo; así también, por parte de la empresa ejecutora, su planificación es ordenada, consistente en la habilitación del campamento base donde se centralizan todas los trabajos, planificación logística de transporte de personal vía aérea (helicópteros) o fluvial, de alimentos y equipos a los campamentos volantes, etc.

Para las planificaciones de ingreso a monitorear las líneas sísmicas, es importante generar un reporte de proyección a los trabajos a realizarse al día siguiente, con el objetivo de insertarse en la logística trazada y el Company Man apoye y solicite la salida al campo para cumplir con las planificaciones.

Así también es importante conocer que la empresa ejecutora (Veritas, en este caso) realiza su planificación de vuelos o traslado vía fluvial, anticipadamente la noche anterior y durante una reunión obligada donde se informa los avances diarios; de esta manera el trabajo se inserta en las actividades normales de la operación sísmica, de ninguna manera se paga vuelo especial para visitar los sitios aperturados.

De no tener esta planificación, lo más probable es que no puedan salir al campo. Esto significa un día perdido y no saber cómo empezar a monitorear. La permanencia en el campamento base solo debe planificarse cuando se necesita hacer el reporte e informar las novedades. Lo recomendable es quedarse y planificar la permanencia en los campamentos volantes desde donde se puede hacer seguimiento directo a los grupos de topografía, taladro o registro. Estos recorridos van marcándose en un plano, así se podrá manejar un recorrido de los diferentes campamentos volantes y líneas sísmicas. Es importante conocer que la permanencia de un grupo de topografía en un campamento es de 7 a 15 días para luego trasladarse al siguiente campamento destinado. Así también hay que tener en cuenta la imposibilidad de recorrer todas las líneas sísmicas o visitar todos los campamentos volantes, por ello las inducciones y reinducciones dadas en el campamento base y en los volantes cumplen una función importante.

\subsubsection{La etapa de topografía}

Antes de iniciar el trabajo debemos analizar los siguientes criterios que permiten a los operadores de sísmica diseñar su estrategia de trabajo: «La ubicación 
y cantidad de campamentos y helipuertos (HP) depende de varios factores como: requerimientos operativos, facilidades de acceso y tipo de equipamiento utilizado en la operación; pero lo más importante es que se ubican cerca de puntos de agua (ríos o quebradas) y se buscan áreas llanas».

Consideramos las variables agua y áreas planas con facilidades de acceso, así también terrazas elevadas pero de relieve plano. Estos son los elementos básicos que todo grupo humano necesita para desarrollarse, recordemos que también lo fue en el pasado.

Por ello es importante que durante los trabajos de monitoreo arqueológico se planifique el ingreso del arqueólogo a los campamentos volantes. Estos pueden estar ubicados cerca de los ríos siendo el traslado vía fluvial, o internados en la selva cuyo ingreso es vía aérea, ingreso que se debe planificar el día anterior con un plan de trabajo en función a la logística programada (aérea o fluvial). Así también, se debe presentar este programa al responsable de la exploración sísmica, en este caso el Company Man. Es importante resaltar que el verdadero trabajo del arqueólogo durante la exploración sísmica está en acompañar a los topógrafos. Paralelo a la habilitación del campamento base, se inicia la etapa de topografía. Hay que tener en cuenta que la logística de movimiento de personal, alimentos y equipos se realiza vía aérea o fluvial.

El traslado del personal a sus respectivos campamentos volantes, sobre todo del grupo de topografía, es inmediato después de recibir las charlas de sensibilidad. Este grupo es quien apertura las líneas sísmicas desde un punto georeferenciado; el avance diario del topógrafo es un tramo de la línea planificada la noche anterior por el equipo de topografía ubicados en el campamento base, quienes por la mañana indican los datos vía radial.

Los campamenteros ubican el área para habilitar los campamentos volantes mediante coordenadas establecidas en el plano, así también el área debe tener ciertas características básicas como por ejemplo: debe estar junto a una quebrada y haber algún espacio para el helipuerto. Aquí se realizan trabajos de desbroce para construir el campamento, ésta es la primera oportunidad de verificar que en cada área desbrozada o cada zanja abierta para letrinas, pozos sépticos o cualquier movimiento de tierra, no contenga material cultural.

Este primer grupo de campamenteros permanece 2 o 3 días, y luego ingresan los grupos de topografía compuesto por 1 topógrafo y 10 a 14 trabajadores, dependiendo de la magnitud del proyecto. En el caso del Lote 88 se ejecutó en 9 meses, habiendo sido programado para 6 meses; así mismo, se abrieron campamentos de apoyo que no se planificaron inicialmente en el plano. 
Dependiendo del tipo de recorrido planificado, pueden ser celdas de tipo panal de abeja o cuadros. Estos grupos topográficos son colocados en sus campamentos volantes, aquí hay que acompañar al topógrafo, pues durante la apertura de las líneas sísmicas cabe la posibilidad de ubicar elementos culturales, además que para el caso de la cerámica muchas veces es difícil reconocer la fragmentería.

Ésta es la etapa más importante del monitoreo arqueológico, los mayores hallazgos se van a registrar aquí. Por ello se realiza el acompañamiento a los diferentes grupos de topografía, durante la apertura de las líneas sísmicas, con el objetivo de verificar en el campo la presencia o ausencia de restos culturales. En este recorrido se monitorean las líneas receptoras y las líneas donde se ubican los puntos de disparo o de salvo, así también se realizan variantes que son rutas ubicadas fuera de las líneas sísmicas, pero que ayudan a buscar rutas más accesibles para que los grupos de las etapas de trabajo siguientes, no tengan mayores problemas de acceso.

En cada cruce de una línea de disparo y una línea receptora se realizan desbroces en un espacio de $4 \times 4 \mathrm{~m}$., conocidos como zonas de descarga (Drop Zone «DZ»). En estos espacios el helicóptero deja material de trabajo (ristras en chinguras). En el campo se identifican estas líneas (receptoras y de salvo) por la numeración con que son asignadas anticipadamente (por ejemplo 6151/stk1646), así también se colocan como señalización cintas en cada estaca dependiendo de la línea (por ejemplo, si es receptora puede ser celeste, blanca, etc. Y si es salvo, la cinta puede ser naranja, roja, etc.) Los DZ también son denominados con letras alfabéticas.

Es importante conocer que todo punto en el terreno está georeferenciado, lo que permite tener la ubicación exacta de los sitios arqueológicos registrados.

Durante las campañas del Lote 88 se registraron 2 sitios arqueológicos, ubicados dentro de la zona de reserva Nahua-Kugapakori; el primero en el «HP 55 el topografo reportó cerámica cuando un trabajador estaba habilitando una escalinata con una palana para que el grupo que se traslade por la línea camine con seguridad, al realizar estos movimientos identificó la cerámica»; el segundo sitio se identificó en «HP 49 cuando el arqueólogo acompañó al grupo de topografía en la apertura de una línea sísmica».

Los demás hallazgos se registraron por los reportes emitidos en la etapa de topografía, como por ejemplo el ubicado en el HP59, «pues un trabajador se acercó a miccionar junto a una piedra y con el líquido comenzó a visualizar unos diseños en forma de estrella, al verificar el lugar efectivamente se trataba de estrellas marinas fosilizadas».

De igual manera en el Lote 56 se registraron 2 sitios arqueológicos, uno ubicado dentro de una chacra y el segundo en el HP 19, esto fue durante el acompañamiento en la etapa de topografía. 
«Es importante también saber que cuando se identifica un sitio durante esta fase, los grupos de topografía pueden seguir realizando su labor de apertura de líneas, pues es la única manera de tener acceso al sitio y poder conocer su dimensión, esto previamente orientado y haciendo un seguimiento más directo a estos grupos de trabajo».

Estos grupos avanzan muy rápidamente, por ello el arqueólogo debe ir verificando e identificando en las líneas qué estacas están cayendo dentro del sitio, a fin de planificar el trabajo durante la fase de perforación y coordinar de manera anticipada el ingreso del arqueólogo.

\subsubsection{Etapa de perforación}

El acompañamiento del arqueólogo en esta etapa de trabajo va a permitir continuar con la verificación de las líneas aperturadas, las cuales no fueron monitoreadas durante la etapa de topografía, pero también hay que tener en cuenta que muchas veces «sobre todo en selva» a simple vista no se visualizan los sitios arqueológicos.

Por ello, hay que planificar primero una reinducción en los campamentos volantes, a estos grupos de trabajo sobre todo, porque ellos utilizan un equipo portátil llamado "carey» para realizar los pequeños orificios de $0.15 \mathrm{~m}$. de diámetro y movimientos de tierra, es allí donde aparece la cerámica que no se visualiza a simple vista en la línea.

Esta etapa también permite anticipar la estrategia a seguir de modo que no se perfore dentro del sitio arqueológico; pero aquí el arqueólogo debe verificar las estacas ubicadas sobre todo en lo que podría ser el límite del sitio, pues el área interna definitivamente queda como zona protegida. Para el caso de los límites es cuando el arqueólogo forma parte del grupo de taladro, eso significa que en cada pozo a perforar se evalúa el avance y se verifica que no contenga cerámica; en caso de aparecer indicios de cerámica el grupo de taladro deja el pozo y pasa al siguiente y así sucesivamente, esto permite al arqueólogo conocer las dimensiones del área con presencia cultural.

Por otro lado los expertos en sísmica (sobre todo registro), aplican los parámetros establecidos por ellos, y el sistema de recuperación de pozos ${ }^{13}$.

Uno de los requisitos es paralizar los trabajos en caso se reporten sitios arqueológicos, lo cual significaría mantener paralizada la operación, hecho que genera problemas graves. Por ello el arqueólogo debe de inmediato realizar estrategias planificadas y coordinadas para dar una solución correcta. Por ejemplo, realizar los recorridos con el apoyo de dos ayudantes (por seguridad nunca se debe caminar solo dentro de la selva, además siempre disponer de una radio de

13 Ver tabla de parámetros de recuperación de datos en anexo 2. 
comunicación), tratar de tener identificado todo el radio que comprende el sitio arqueológico e identificar qué estacas se ubicaron dentro del sitio, pueden ser fuentes o receptoras.

Cuando las estacas corresponden a líneas receptoras no es mayor problema, pero si las estacas son de salvo hay que retirarlas fuera del área arqueológica, en coordinación con los jefes y responsables de esta etapa de trabajo, para que todo el equipo correspondiente solucione el inconveniente y el registro de la sísmica no se altere. Como mencioné anteriormente, para el caso de quebradas, fuentes de agua, viviendas, los expertos tienen parámetro de recuperación de datos, los cuales también se aplican en caso de sitios arqueológicos. Para ello hay que asegurarse y tener identificada el área correspondiente al sitio y conocer las estacas ubicadas dentro del sitio para ser recuperadas por ellos en otros lugares. Para el caso de los límites del sitio identificado hay que hacer la perforación con la compañía del arqueólogo para que verifique cuál es el límite de la zona identificada y no haya más evidencias arqueológicas.

Se han registrado evidencias culturales cuando se realizó la apertura de una pequeña cuneta de $0.40 \times 0.40 \mathrm{~m}$, la cual sirve para dar estabilidad al equipo de perforación o taladro. Al realizar este pequeño movimiento de tierra, la cerámica comienza a salir a flote.

Para colocar el carey se realiza un pequeño movimiento de tierra, allí es importante estar presente, porque muchas veces no fue posible verificar las evidencias culturales en la primera etapa y también se tiene que recorrer las líneas con el grupo de perforación; por ejemplo, durante la campaña de sísmica del Lote 56 se realizó la identificación del sitio numero 000 en el cual al realizar una pequeña zanja de unos 50×50x0.30 comenzó aparecer cerámica, hecho que motivó una evaluación, el sitio quedó reservado para realizar excavación arqueológica.

Otro ejemplo que vale la pena que se conozca es cuando se ubicó el sitio arqueológico del HP 55, además de trabajar directamente con el grupo de topografía, realizándose pequeños sondeos para verificar la dimensión del área, durante la etapa de perforación fue necesario acompañar a los grupos que están en el área para poder verificar que donde se perfore y se coloque el explosivo se realice fuera del área con material cultural.

\subsubsection{Etapa de registro}

El equipo de registro ingresa cuando el grupo de perforación avanzó la cuarta parte del lote. Aquí ingresan los regadores de cables, quienes colocan además los geófonos en cada estaca determinada.

Esta etapa de trabajo es importante para el arqueólogo, porque va permitir verificar las líneas que no fueron monitoreadas durante las etapas anteriores, así 
también hay que coordinar charlas de reinducción en los campamentos volantes donde se ubican los diferentes grupos, pues lo que no se pudo observar en las etapas anteriores, cabe la posibilidad que en esta etapa se pueda registrar alguna evidencia cultural, cuando los regadores incrustan los geófonos bajo tierra.

Dependiendo del tipo de sísmica también es la magnitud de la estrategia a desarrollar. En caso de una sísmica 3D, donde las líneas de salvo con registro forman un plano cartesiano, y el sitio caiga en la línea de registro, el arqueólogo no se preocupa mucho en que se deteriore el sitio, pues los geófonos -incrustados bajo tierra- no afectan. Pero hay que tener mucha precaución durante el plan de abandono, pues inmediatamente que culmina esta etapa hay un equipo que se encarga de cerrar los campamentos volantes con material orgánico en superficie.

En esta etapa de trabajo se registró un sitio arqueológico en el Lote 88, ahora lo conocemos como Maniroriato y varios hallazgos en el Lote 56.

\section{VII. ¿Qué estrategia emplear cuando se tiene que trabajar dentro de centros poblados como las comunidades nativas?}

Son importantes los datos de ubicación de los campamentos y helipuertos con respecto a la propiedad del territorio de las comunidades, porque en función a ello se determina la prioridad del monitoreo. Para los que se ubican cerca al río, puesto que en esas áreas hay mayor ocupación por parte de las comunidades, se realizaron reuniones informativas y de consulta con los jefes de cada comunidad, para no afectar chacras y sitios ceremoniales históricos, como por ejemplo cementerios. Todos los trabajos se realizaron de mutuo acuerdo con las comunidades, los que constan en actas de reunión firmadas.

Esta estrategia es importante, porque la etapa de exploración sísmica también abarca áreas de chacra, lo que permite ingresar a monitorear estas áreas de cultivo. Aquí hay que poner mucha atención porque éstas se ubican siempre siguiendo el patrón establecido, áreas planas y con fuentes de agua. Los pobladores al remover la tierra para sembrar encontraron instrumentos líticos. Esto se pudo constatar por los datos de informantes y también durante el monitoreo en el Lote 56, donde se ubicó el hallazgo de un instrumento lítico dentro de una chacra, así también se ubicaron otros instrumentos dentro de la comunidad cuando los pobladores hacían remoción de tierra para construir sus viviendas.

Es importante tener en cuenta que para el caso de centros poblados, siempre hay que coordinar con el jefe de la comunidad y las autoridades, para poder realizar los cateos exploratorios y así tener un reporte detallado del sitio. 


\section{VIII. ¿Cómo trabajar en caso se ingrese a zonas de reserva territorial?}

Todos conocemos que el trabajo dentro de una zona de reserva es sumamente delicado, si bien es cierto que los grupos en las diferentes etapas de trabajo en sísmica respetan parámetros estrictos cuando se ingresa a estas zonas, el arqueólogo debe seguir con todas las normas establecidas por la empresa. Hay que adicionar un plan de contingencia antropológico para poblaciones en contacto inicial, pues cabe la posibilidad de encontrarse con pobladores catalogados dentro de estos grupos. Este plan también es avalado por el gobierno, pues se ajusta al sistema de normas legales que tiene el Estado peruano para poblaciones indígenas.

Por ejemplo para el caso del sitio HP 55, fue necesario ingresar a evaluar el área, sin embargo también había que seguir con las pautas establecidas por la empresa para poblaciones en contacto inicial. Los requisitos consisten en tener dos guías preferentemente, uno situado delante del grupo y otro al final del mismo. En caso de encontrarse con nativos de esta categoría, solamente el guía realiza la comunicación.

El sitio HP 55 quedó marcado como zona intangible, porque no solamente aquí es necesario la aceptación del INC, sino también debe emitir una autorización. El ingreso para excavar en esta zona de reserva, implica preparar una logística particular. Para excavar hay que adicionar la autorización del estado y si se pretende realizar excavaciones hay que asegurarse de seguir las instrucciones del plan de contingencia antropológico.

\section{¿Cómo identificar sitios arqueológicos en zona de Selva?}

Cada etapa de trabajo tiene una estrategia apropiada y una logística la cual se debe aprovechar, sin tener que emplear un recurso adicional y así lograr resultados eficientes. Así también, en esta zona la ubicación de los sitios arqueológicos tienen características apropiadas a los cuales podríamos llamar patrón de asentamiento.

Este patrón lo definimos como: «Terrazas elevadas, de relieve plano, en algunos casos tiene vegetación secundaria correspondiente a pacales, así también se ubican junto a ríos, quebradas o puntos con agua».

\section{Estrategia de trabajo para la etapa de construcciones}

La etapa de construcción comprende las plataformas y áreas donde se realizarán las plataformas para los pozos y toda etapa que corresponde a las obras civiles, esto se desarrolla en la plataforma donde se realizan: 
- Zanjeo para cableado eléctrico

- Zanjeo para el campamento base

- $\quad$ En algunos casos se proyecta un aeródromo

\section{Estrategia en linea de flow line}

Durante esta etapa, la importancia del monitoreo arqueológico consiste en hacer un seguimiento diario de la apertura de la línea donde se coloca la tubería para trasportar el gas y los hidrocarburos de los pozos a la planta de gas. El seguimiento a todo este movimiento de tierra se realizó paralelo a la etapa de construcciones, así como el seguimiento al cableado para electricidad. Esto implica que el arqueólogo debe estar permanentemente con el equipo de maquinaria que ingresa a abrir las línea de conducción, apertura de un ancho de 18 metros donde se realiza el zanjeo de toda la línea por donde se coloca la tubería que conduce el gas.

Es importante mencionar que durante esta etapa se excavó el sito arqueológico de Maniroreato, también se registraron hallazgos de cerámica y utensilios de piedra en el Lote 88. 


\section{Anexo}

1. Registro Fotográfico por etapas de trabajo

- $\quad$ Etapa de topografía

- Etapa de perforación

- $\quad$ Etapa de registro

2. Parámetros de medidas para recuperación de pozos no perforados

Cuadro: Recuperación de datos

\begin{tabular}{ll}
\hline Caños & 15 Metros \\
\hline Quebradas & 30 Metros \\
\hline Centro Poblado & 180 Metros \\
\hline Cementerio & 80 Metros \\
\hline Sitio Arqueológicos & 80 Metros \\
\hline
\end{tabular}

- $\quad$ Tabla utilizada por la empresa que realizó la exploración sísmica del Lote 88 y Lote 56 VERITAS DGC LANG como estrategia de recuperación de datos, cuando las estacas se ubican en puntos de caños, quebradas, centros poblados, cementerios, sitios arqueológicos, se respeta esta distancia y se ubica el punto en otro punto para no perder la información.

- $\quad$ Otra empresa que maneja esta tabla de recuperación es South America Exploration.

- Desconozco si estas medidas son utilizadas por otras empresas de exploración sísmica.

3. Cuadro de Hallazgos de Lote 88 y Lote 56

\begin{tabular}{lll}
\hline Sitio 1 Niateni - Kovantiani & 6 & Varíos asentamientos \\
\hline Sitio Camisea 2 & 1 & Un asentamiento \\
\hline Sitio Camisea 3 & 2 & Dos asentamientos \\
\hline Sitio 4 Manirorato & 1 & Un asentamiento \\
\hline Sitio 5 Pucacuro & 1 & Posibilidad de 2 asentamientos \\
\hline Sitio 6 Miaria & 1 & Un asentamiento \\
\hline Total : 6 Sitios & 9 & \\
\hline
\end{tabular}

Fuente: Proyecto Camisea, Lote 88. 


\section{Agradecimientos:}

A las comunidades Machiguenga y Yines, al departamento de Asuntos Comunitarios, a la gerencia de EHS y CCNN de PLUSPETROL Perú Corporation S.A.; al especialista en operaciones de adquisición de datos sísmicos Ing. René Aguilar, Company Man de la Operación Símica del Lote 56, quien orientó al equipo en las explicaciones técnica; Malaquías Lizardo Murayari, mi amigo con quien aprendimos mutuamente (Comunidad Nativa de Bufeo Pozo del grupo étnico Yine).

\section{Bibliografía}

\section{PLUSPETROL PERU CORPORATION}

2001 Estudio de Impacto Ambiental del Lote 88, Camisea y Área de Influencia Estudio deImpacto Social. Elaborado por la empresa ERM.

\section{PLUSPETROL PERU CORPORATION}

2005 Estudio de Impacto Ambiental del Lote 88, Camisea y Área de Influencia Estudio de Impacto Social. Elaborado por la empresa ERM. 